\title{
Efficacy of Low Dose Naltrexone in Psoriasis
}

\author{
Sidra Khan, Rabia Ghafoor and Sana Kaleem \\ Department of Dermatology, Jinnah Postgraduate Medical Centre, Karachi, Pakistan
}

\begin{abstract}
Objective: To determine the efficacy of low dose naltrexone in the patients of psoriasis.

Study Design: Non-randomised clinical trial.

Place and Duration of Study: Department of Dermatology, Jinnah Postgraduate Medical Centre, Karachi from January to July 2019.

Methodology: All patients of mild, moderate, and severe psoriasis with age above 13 years without any comorbids were included in this study. Patients were started on tablet naltrexone $6 \mathrm{mg}$ daily after assessing PASI, BSA and DLQI scores and were called every month for follow-up. After three months of treatment, these scores were assessed again. SPSS 23 was used to analyse the data.

Results: Out of 71 patients, $37(52 \%)$ were males and $34(48 \%)$ were females with age ranging from 13 to 60 years with mean age of $37.85 \pm 12.211$ years. The mean duration of disease was $5.27 \pm 3.084$ years. Before treatment $1(1.4 \%), 20(28.2 \%), 24$ (33.8\%), 26 (36.6\%) patients had mild, moderate, severe and very severe psoriasis according to PASI score. After treatment, 14 $(19.7 \%), 23(32.4 \%), 23(32.4 \%), 11(15.5 \%)$ patients had mild, moderate, severe and very severe psoriasis. The mean PASI, BSA and DLQI were $18.47 \pm 8.157,11.97 \pm 3.873$ and $22.63 \pm 5.235$ before treatment, respectively. The mean PASI, BSA and DLQI were $13.51 \pm 8.017,8.07 \pm 3.650$ and $16.31 \pm 7.056$ after treatment, respectively.

Conclusion: Low dose naltrexone is an effective therapy for psoriasis as in other chronic dermatological diseases. It is a costeffective therapy with few tolerable side-effects. It requires further studies for long-term response.
\end{abstract}

Key Words: Naltrexone, Psoriasis, PASI, DLQI, BSA.

How to cite this article: Khan S, Ghafoor R, Kaleem S. Efficacy of Low Dose Naltrexone in Psoriasis. J Coll Physicians Surg Pak 2020; 30(06):579-583 https://doi.org/10.29271/jcpsp.2020.06.579.

\section{INTRODUCTION}

Psoriasis is a chronic T-cell mediated disorder. The activated T-cell secretes cytokines that result in keratinocytes hyperproliferation and abnormal differentiation. These cytokines result in chemotaxis of neutrophils and lymphocytes in skin. ${ }^{1}$ It is a multi-factorial disease. Individual has genetic susceptibility for psoriasis, but environmental factors trigger the disease like infections, drugs, cold, trauma and stress. ${ }^{2}$ It is a multi-system disease but mainly affects skin, nails, and joints. In its most common form, it presents as salmon pink plaques, covered with thick silver scales, mostly on the scalp, trunk, buttocks, and external surfaces of the limbs. On removal of successive layers of scales from papules and plaques of psoriasis, there are pinpoint bleeding points called as Auspitz sign. This disease impacts adversely on patients' quality of life, causes embarrassment, limitation in the choice of clothing and psychological distress which further aggravates it. It impacts economically due to off days from work as well. ${ }^{3}$

Correspondence to: Dr. Sidra Khan, Department of Dermatology, Jinnah Postgraduate Medical Centre, Karachi, Pakistan

E-mail: dr.sidrakhan786@gmail.com

Received: February 20, 2020; Revised: June 08, 2020;

Accepted: June 17, 2020

DOI: https://doi.org/10.29271/jcpsp.2020.06.579
There are various treatment modalities, which include topical and systemic therapy as well as phototherapy; but each of them carry their own side effects and contraindications. One the most attractive emergent therapies nowadays is low dose naltrexone (LDN), which has analgesic, antipruritic as well as anti-inflammatory properties and is used for various other dermatological disorders like Hailey-Hailey disease, Lichen planopilaris and Crohn's disease. ${ }^{4}$

There is no clinical trial for role of low dose naltrexone in psoriasis; previously a few case reports documented the effectiveness of LDN in plaque and erythrodermic psoriasis.

The objective of this study was to determine the effectiveness of low dose naltrexone in psoriasis based on psoriasis area severity index (PASI), body surface area (BSA) and dermatology life quality index (DLQI).

\section{METHODOLOGY}

This prospective non-randomised clinical trial was conducted in the Department of Dermatology, Jinnah Postgraduate Medical Centre, Karachi from January to July 2019 after receiving approval from the institutional ethical and research committee of the hospital. It has been registered with international RCT registry (ClinicalTrials.gov Identifier NCT04250792). The sample size was calculated through NCSS software. 


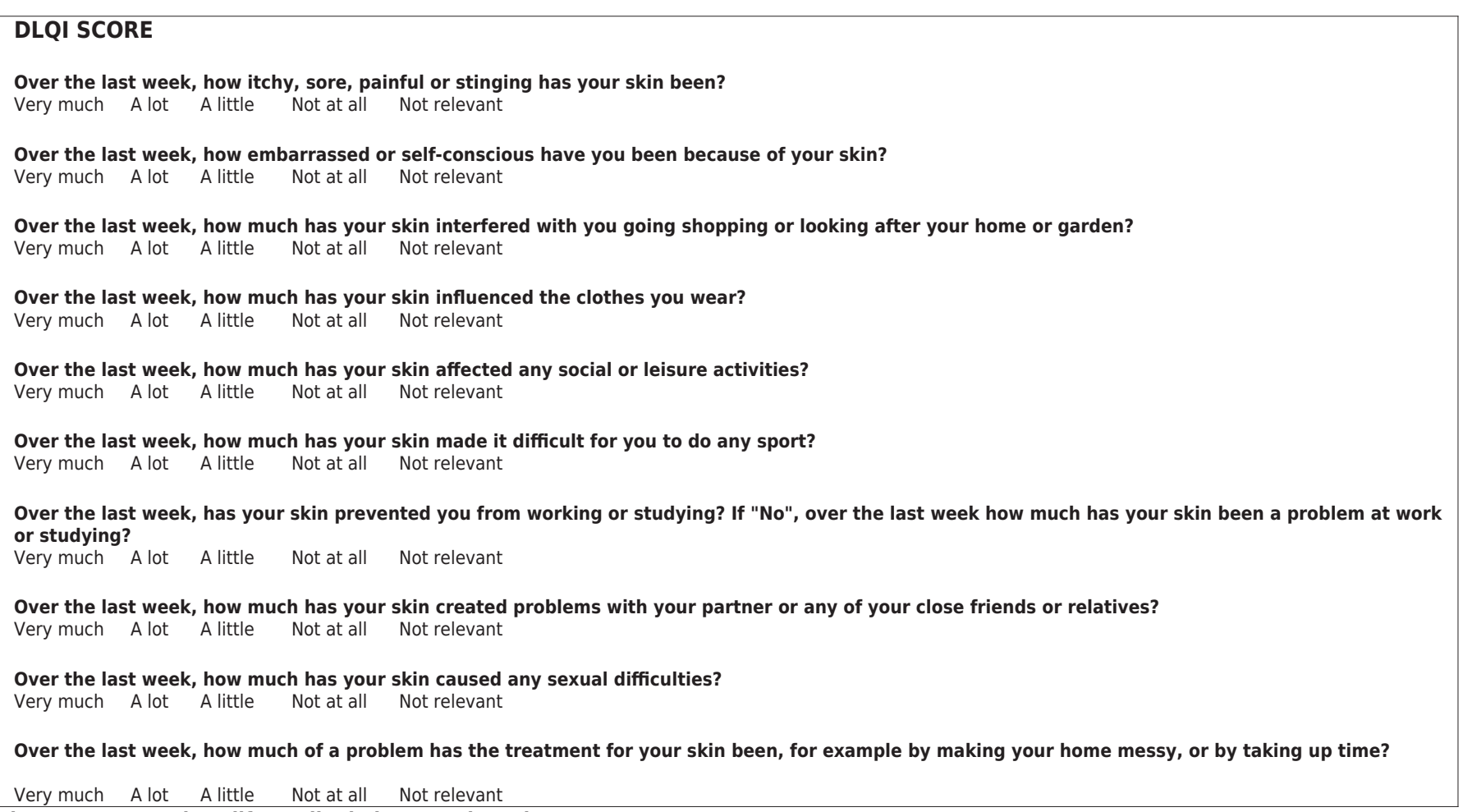

Figure 1: Dermatology life-quality index questionnaires.

From patients referred to Department of Dermatology, 71 patients were selected by purposive method of non-probability sampling. Seventy-one diagnosed patients of plaque psoriasis of either gender were included in this study, in which 70 patients were without arthritis while one patient was known case of psoriatic arthritis, with age ranging from 13-60 years after excluding hypertension, cardiovascular disorders, pregnancy, lactation and hypersensitivity to naltrexone. The procedure was explained to each patient and written informed consent was taken. Before starting low dose naltrexone treatment, psoriasis area and severity index (PASI), body surface area (BSA) and dermatology life quality index (DLQI) were calculated. Each patient was prescribed tablet naltrexone $6 \mathrm{mg}$ daily after checking all baseline investigations of complete blood counts, renal profile, and liver function tests. Patients were asked to follow up each month for a period of three months to observe patients' compliance and side effects of treatment; and at the end of three months from baseline PASI, BSA and DLQI scores were calculated to observe the disease activity and effectiveness of treatment.

PASI is an index, used to measure the severity and extent of psoriasis. ${ }^{5}$ It is measured in terms of intensities of erythema, induration and scaling of lesion on head and neck (A1), upper limbs (A2), trunk (A3) and lower limbs (A4) as none (0), mild (1), moderate (2), severe (3) or very severe (4). After adding all intensities for each separated part of the body, they are multiplied by $0.1,0.2,0.3$, and 0.4 , respectively. Then scores are multiplied with body area score which is calculated as nil (0), $1-9 \%(1), 10-29 \%(2), 30-49 \%(3), 50-69 \%(4), 70-89 \%(5)$ and $90-100 \%(6)$. After adding all parameters of scoring system, the final scores are obtained.
BSA is defined as percent of total body surface area affected with disease, where patient's own single handprint represents $1 \%$ of $\mathrm{BSA} .{ }^{6}$ It is a convenient and rapid method of estimating the extent of body surface area affected. DLQI is a simple questionnaire that consists of 10 questions as illustrated in Figure 1, to assess the impact of psoriasis and its treatment on daily activities and the impact on the social behaviour of the patient towards their friends and relatives. ${ }^{7}$

The qualitative variables were expressed by all these scores, w.r.t the ranges as mild, moderate, severe, and very severe. These were expressed as frequency along with percentages of patients according to the severity of disease and its effect on the quality of life. PASI score was interpreted as patients had mild, moderate, severe and very severe disease according to the range of final scores as $0-5,6-12,13-20$ and $>20$, respectively. According to BSA, the disease was categorised as mild, moderate and severe if score was $<3 \%, 3-10 \%$, and $>10 \%$ respectively. ${ }^{8}$ DLQI scores were interpreted, regarding the effect on patient's life as 0-1 (No effect), 2-5 (small effect), 6-10 (moderate effect), 11-20 (very large effect), 21-30 (extremely large effect). Qualitative data was subjected to analysis through chi-square test. Quantitative variables were expressed as mean \pm standard deviation (SD) of the scores and analysed with paired t-test. The statistical analysis was done through IBM SPSS statistics software version 23 . The level of significance was set at $p<0.05$. The side effects were also assessed regarding nausea, headache, decreased appetite, dizziness, palpitation, liver enzymes level, renal and complete blood profiles.

\section{RESULTS}

In this study, 71 patients were enrolled. The mean age was 
$37.85 \pm 12.21$ years. There were 37 male (52\%) and 34 female (48\%) patients. The mean duration of disease was $5.27 \pm 3.08$ years.

According to PASI score, 1 (1.4\%) patient had mild, 20 (28.2\%) patients had moderate, 24 (33.8\%) patients had severe and 26 $(36.6 \%)$ patients had very severe disease. After three months of treatment from baseline, 14 (19.7\%) patients had mild, 23 $(32.4 \%)$ patients had moderate, $23(32.4 \%)$ patients had severe and $11(15.5 \%)$ patient had very severe disease. Chi-square = $50.888^{a}, p<0.001$.

According to BSA score, 29 (40.8\%) patient had moderate and $42(59.2 \%)$ patients had severe disease. After three months of treatment from baseline, 7 (9.9\%) patient had mild, 49 (69.0\%) patients had moderate and $15(21.1 \%)$ patients had severe disease. Chi-square $=13.395^{\mathrm{a}}, \mathrm{p}<0.001$.

The dermatology life quality index (DLQI) scores before treatment showed that $29(40.8 \%)$ patients had very large effects and $42(59.2 \%)$ patients had extremely large effects of disease and its treatment on their daily activities and social behaviours. After three months of treatment from baseline, it showed 3 (4.2\%) patients had small effects, 14 (19.7\%) patients had moderate effects, $29(40.8 \%)$ patients had very large effects and $25(35.2 \%)$ patients had extremely large effects of disease and its treatment on their daily activities and social behaviours. Chi-square $=28.158^{\mathrm{a}}, \mathrm{p}<0.001$.

Table I: Results of paired sample test.

\begin{tabular}{|c|c|c|}
\hline Parameters & Mean \pm SD & $\begin{array}{l}\text { Paired samples } \\
\text { test (sig) }\end{array}$ \\
\hline PASI before treatment & $18.47 \pm 8.157$ & \multirow{2}{*}{$\begin{array}{l}t=6.917 \\
p<0.001\end{array}$} \\
\hline PASI after treatment & $13.51 \pm 8.017$ & \\
\hline DLQI before treatment & $22.63 \pm 5.235$ & \multirow{2}{*}{$\begin{array}{c}t=10.262 \\
p<0.001\end{array}$} \\
\hline DLQI after treatment & $16.31 \pm 7.056$ & \\
\hline BSA before treatment & $11.97 \pm 3.873$ & \multirow{2}{*}{$\begin{array}{l}\mathrm{t}=8.061 \\
\mathrm{p}<0.001\end{array}$} \\
\hline BSA after treatment & $8.07 \pm 3.650$ & \\
\hline
\end{tabular}

The mean scores were significantly lower after treatment. The mean difference was $4.963 \pm 6.046$ for PASI, $3.901 \pm 4.078$ for BSA and $6.324 \pm 5.193$ for DLQI; confidence level set at $95 \%$, with confidence interval of 3.532 - 6.394, 2.936 - 4.867 and 5.095 - 7.553 for PASI, BSA and DLQI respectively. Results are illustrated in Table I.

The most common side effects observed in this study were nausea, headache, insomnia, and anxiety which were managed symptomatically, tolerance developed after three doses of LDN and side effects disappeared thereafter.
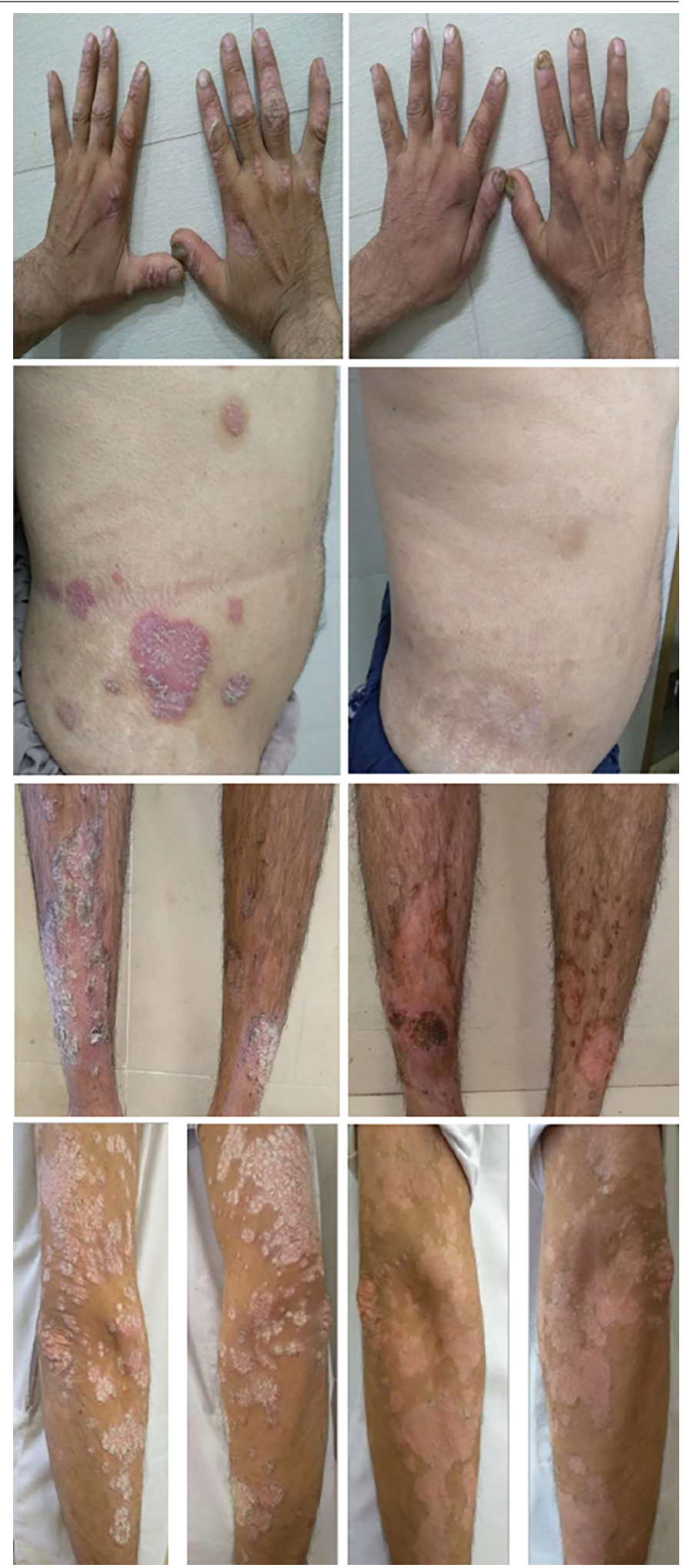

images show improvement with LDN.

\section{DISCUSSION}

Psoriasis is a chronic inflammatory disease with relapsing and remitting in nature. The hallmark of disease is the sustained inflammatory process that results in an increase in the turnover of keratinocytes. The process of cornification occurs in 7 days in 
psoriasis, instead of 30-45 days in a healthy skin. ${ }^{9}$ It is a multi-factorial disease. One-third of cases of psoriasis have positive family history. HLACw6 is strongly associated with early disease and arthropathy. ${ }^{10}$ In psoriasis, dysregulation of skin immune system is suggested due to its association with HLA antigens, chronicity, relapse, and remission of the disease with immunosuppressive drugs. ${ }^{11}$ It is a T-cell driven disease mainly involving Th1 and Th17 cells. They play a pivotal role through stimulation of cytokines secretion, like IL-6, IL-7, IL-22, INF- $\gamma$ and TNF- $\alpha{ }^{12}$ In turn, these cytokines stimulate proliferation in keratinocytes, angiogenesis, and recruitment of inflammatory cells.

Treatment approaches are based on severity of disease. In turn the severity of disease depends on extent of disease, sites of body involved, degree of inflammation, response to treatment and impact of disease on the quality of life. It can be assessed through different scoring system for example Psoriasis Area Severity Index (PASI), Body Surface Area (BSA), Physician Global Assessment (PGA), Dermatology Life Quality Index (DLQI). Rule of ten defines a severe psoriasis if anyone of the absolute score is more than $10 .{ }^{13}$ Patients with BSA $<10 \%$ have greater risk for clinical comorbidities, but when BSA $>10 \%$, it is associated with high mortality rate. ${ }^{14}$ Systemic therapy is also required as well, when there is only exposed parts are involved because it has severely impaired the quality of life of the patients due to functional impairment and disfiguring that results in psycho-social distress and embarrassment, further aggravating the disease. Treatment approaches can be categorised into topical, systemic and phototherapy. Topical therapy is an effective approach in mild cases, but it is inconvenient to apply for prolong periods. ${ }^{15}$ For moderate to severe disease, combination therapies are required. Systemic therapy is required when PASI $>10$, PASI $<10$ with scalp, face, hands, nails, palmoplantar, genital disease, disease unresponsive to topical treatment, DLQI >10, and active psoriatic arthritis. Palmoplantar disease is associated with severe functional disability and impairment in quality of life. ${ }^{16}$ Due to chronic disfiguring disease, patient develops depression, anxiety, social isolation and suicidal ideas, it further aggravates the severity, duration and recurrences of disease. ${ }^{17}$ The interval between recurrences may vary from months to years. Remission is achieved by long term control of the disease.

Naltrexone and its active metabolite 6 - $\beta$ naltrexol are the reversible competitive antagonists at $\mu$ and $k$ receptors. Low dose naltrexone (LDN) transiently blocks opioid receptors and plays an important role in control of cell growth and inflammatory processes by increasing the levels of endogenous opioids through positive feedback mechanisms, for example, endorphins and enkephalins. These peptides promote healing, inhibit cell growth, and reduce inflammation. ${ }^{18}$ LDN also increases the production of opioid growth factors and their receptors through positive feedback mechanism at the translational level. They modulate cell growth by their action on G1/S phase through p16 and p21 cyclin dependant inhibitory kinases. LDN blocks toll like receptors (TLRs) signalling that blocks the release of pro-inflammatory cytokines like IL-6, IL-12, TNF and NFKB. It modulates T and $B$ lymphocytes and shifts immune response from Th2 to Th1. TLRs play role in the innate immune system of the body. ${ }^{19}$
These receptors are expressed on macrophages, dendritic cells, and microglia. Infections and inflammations result in upregulation of the receptors on these cells, in turn which causes a cascade of pro-inflammatory cytokines.

In this study, patients treated with low dose naltrexone had improved their psoriasis within three months of treatment and the patient with psoriatic arthritis had improved with prolong therapy of four months as illustrated in Figure 2. The efficacy of LDN was more pronounced in guttate and annular psoriasis. Response of therapy was most significant in female patients. The patient who was known case of psoriatic arthritis showed no significant reduction in disease activity despite of being on methotrexate for one year, was started treatment with LDN; and after four months of treatment, he had significant reduction in his PASI score from 20 at the start of treatment to PASI score of 1 with the treatment. He stopped all analgesics by himself as he felt improvement in his arthralgia. LDN results in the upregulation of $\beta$-endorphins. These $\beta$-endorphins have great role in relieving joint pain along with its anti-inflammatory properties. ${ }^{20}$ While on LDN, patients required emollients less frequently. It improved their quality of life because frequent application of emollients was causing stinging of clothes and difficulty in daily activities of life. Control of disease activity was not consistent in all patients, as with other treatment modalities. Some patients did not improve, and they were considered as non-responder to the treatment. It may be due to mutation in $\mu$ receptors or differences in endogenous pain and inflammatory modulation system. ${ }^{21}$

Treatment goals in psoriasis are based on the reduction of erythema, scaling and thickness of plaques as assessed with PASI, BSA, improvement of quality of life and reduction in exacerbations and completely get rid of lesions. Treatment response is generally evaluated after three to four months of therapy. If response is not achieved, dose can be increased, or combination therapy can be given. If there is a failure of reduction of at least $50 \%$ of PASI score, it will be considered as non-responder to the treatment. ${ }^{22}$

Therapeutic choices are continuously changing with the approval of new drugs. The long-term use of systemic therapy results in poor tolerability and cumulative toxicity like liver toxicity from methotrexate, renal toxicity from cyclosporine, skin carcinogenesis from phototherapy, infections and production of anti-drugs antibodies associated with biologics. ${ }^{23}$ Whereas, treatment with low dose naltrexone is free from severe adverse effects of liver toxicity, renal damage, carcinogenesis and infections. Naltrexone has half-life of four hours, metabolised in its active compound $6-\beta$ naltrexol which has half-life of 13 hours and then excreted mainly in urine. ${ }^{24}$ Treatment with LDN significantly reduces the need for analgesics, antipruritic and DMARDs as observed during this study. It is a cost-effective therapy with very few tolerable side effects.

Major limitation of this study is that it has not compared the response of low dose naltrexone with other treatment modalities. Large scale multicentric studies are required to further validate the drug. 


\section{CONCLUSION}

Treatment of psoriasis with low dose naltrexone is an emergent cost-effective therapy with well tolerable side effects profile. It requires further cohort studies to observe its role in the reduction of flares of disease and psoriatic arthritis, and durability of its effect for prolong disease control.

\section{ETHICAL APPROVAL:}

Legal and ethical approvals were obtained prior to initiation of the research work from institutional review board committee of Jinnah Postgraduate Medical Centre, Karachi.

\section{PATIENTS' CONSENT:}

Informed consents were obtained from patients to publish the data concerning their case.

\section{CONFLICT OF INTEREST:}

Authors declared no conflict of interest.

\section{AUTHORS' CONTRIBUTION:}

SK: Contributed to the design research, ethical approval, patient consent, data collection, analysis of the results and writing of the manuscript.

RG: Involved in supervising the work.

SK: Provided critical feedback.

\section{REFERENCES}

1. Chiang CC, Cheng WJ, Korinek M, Lin CY, Hwang TL. Neutrophils in psoriasis. Front Immunol 2019; 10:2376.

2. Alharbi T, Alzahrani A, Hakami A, Almohammadi A, Alsaadi A, Magbel NB, et al. Psoriasis pathophysiology and impact on life. Intern J Commun Med Public Health 2018; 5(9):3663-7.

3. Anderko M, Triviño FJ, Sharples CL. Calcipotriol plus betamethasone dipropionate aerosol foam for scalp psoriasis. Clin Cosmet Investig Dermatol 2019; 12:699-705.

4. Sonthalia S, Agrawal M, Talwar A, Goldust M. Low-dose naltrexone-induced remission in Hailey-Hailey disease maintained in remission with topical combination of ketamine and diphenhydramine. Indian Dermatol Online J 2019; 10(5): 567-70.

5. Rigas HM, Bucur S, Ciurduc DM, Nita IE, Constantin MM. Psychological stress and depression in psoriasis patients - A dermatologist's perspective. Mædica (Buchar) 2019; 14(3): 287-91.

6. Walsh JA, Jones $H$, Mallbris L, Duffin KC, Krueger GG, Clegg $\mathrm{DO}$, et al. The physician global assessment and body surface area composite tool is a simple alternative to the psoriasis area and severity index for assessment of psoriasis: Post hoc analysis from pristine and presta. Psoriasis (Auchkl) 2018; 8:65-74.

7. Chernyshov PV. The evolution of quality of life assessment and use in dermatology. Dermatology 2019; 235(3): 167-74.
8. Kim WB, Jerome D, Yeung J. Diagnosis and management of psoriasis. Canadian Family Physician 2017; 63(4):278-85.

9. Roberson ED, Bowcock AM. Psoriasis genetics: Breaking the barrier. Trends in Genetics 2010; 26(9):415-23.

10. Benhadou F, Mintoff D, Del Marmol V. Psoriasis: Keratino-cytes or immune cells-which is the trigger? Dermatology 2019; 235(2):91-100.

11. De Keyser E, Busard Cl, Lanssens S, Meuleman L, Hutten BA, Costanzo A, et al. Clinical consequences of antibody formation, serum concentrations, and HLA-Cw6 status in psoriasis patients on ustekinumab. Therapeutic drug monitoring 2019; 41(5):634-9.

12. Budamakuntla L, Shree-Lakshmi HV, Bansal A, Venkatarayaraju SK. Spotlight on itolizumab in the treatment of psoriasis - Current perspectives from India. Psoriasis (Auchkl) 2019; 9:19-27.

13. Strober $B$, Ryan $C$, van de Kerkhof $P$, van der Walt J, Kimball $A B$, Barker J, et al. Recategorization of psoriasis severity: Delphi consensus from the International Psoriasis Council. J Am Acad Dermatol 2020; 82(1):117-22.

14. Noe MH, Shin DB, Wan MT, Gelfand JM. Objective measures of psoriasis severity predict mortality: A prospective population-based cohort study. J Invest dermatol 2018; 138(1): 228-30.

15. Martin G, Young M, Aldredge L. Recommendations for initiating systemic therapy in patients with psoriasis. J Clin Aesthetic Dermatol 2019; 12(4):13-26.

16. Miceli A, Schmieder GJ. Palmoplantar psoriasis. Stat Pearls Publishing; 2019.

17. Liluashvili S, Kituashvili T. Dermatology Life Quality Index and disease coping strategies in psoriasis patients. Postepy Dermatol Alergol 2019; 36(4):419-24.

18. Zashin S. Sjogren's syndrome: Clinical benefits of low-dose naltrexone therapy. Cureus 2019; 11(3):e4225.

19. Rendon A, Schäkel K. Psoriasis pathogenesis and treatment. Intern J Molecul Scien 2019; 20(6):1475-503.

20. Monasterio EP. Low-dose naltrexone: An alternative treatment for erythrodermic psoriasis. Cureus 2019; 11(1): e3943.

21. Burton EF, Suen SY, Walker JL, Bruehl S, Peterlin BL, Tompkins DA, et al. Ethnic differences in the effects of naloxone on sustained evoked pain: A preliminary study. Divers Equal Health Care 2017; 14(5):236-42.

22. Mrowietz U, Kragballe K, Reich K, Spuls P, Griffiths CE, Nast A, et al. Definition of treatment goals for moderate to severe psoriasis: A european consensus. Arch Dermatol Res 2011; 303(1):1-10.

23. Liau MM, Oon HH. Therapeutic drug monitoring of biologics in psoriasis. Biologics 2019; 13:127-32.

24. Toljan K, Vrooman B. Low-dose naltrexone (LDN) - Review of therapeutic utilisation. Med Sci (Basel) 2018; 6(4):82. 